\title{
Time dependent beam focusing at the DARHT-II injector diode.
}

\author{
S. Eylon, E. Henestroza, W. Fawley, S. Yu \\ Lawrence Berkeley National Laboratory, Berkeley, CA 94720 USA
}

Abstract

The injector for the second axis of the Dual-Axis Radiographic Hydrotest Facility (DARHT) is being designed and constructed at LBNL. The injector consists of a single gap diode extracting $2 \mu \mathrm{s}, 2 \mathrm{kA}, 3.2 \mathrm{MeV}$ electron beam from a 6.5 inches diameter thermionic dispenser cathode. The injector is powered through a ceramic column by a Marx generator. We also investigated the possibility of extracting a beam current of $4 \mathrm{kA}$.

The focusing system for the electron beam consists of a Pierce electrostatic focusing electrode at the cathode and three solenoidal focusing magnets positioned between the anode and induction accelerator input. The off-energy components (beam-head) during the $400 \mathrm{~ns}$ energy rise time are overfocused, leading to beam envelope mismatch and growth resulting in the possibility of beam hitting the accelerator tube walls.

The anode focusing magnets can be tuned to avoid the beam spill in the $2 \mathrm{kA}$ case. To allow beam-head control for the $4 \mathrm{kA}$ case we are considering the introduction of timevarying magnetic focusing field along the accelerator axis generated by a single-loop solenoid magnet positioned in the anode beam tube.

We will present the beam-head dynamics calculations as well as the solenoid design and preliminary feasibility test results.

\section{INTRODUCTION}

A high voltage, high current, long pulse and high quality electron beam injector for a linear induction accelerator for flash-radiography applications is being developed at LBNL for the second axis of the Dual-Axis Radiographic Hydrotest Facility (DARHT). The injector conceptual design is based on LBNL Heavy Ion Fusion Injector technology [1], and beam dynamics simulations. Figure 1 shows the main components of the $3.2 \mathrm{MV}$ electrostatic injector assembly. The injector is driven by a Marx pule generator. The marx output pulse is fed through a $3.2 \mathrm{MV}$ insulating graded (ceramic) column into the beam diode.

\section{THE 3.2 MV ELECTRON DIODE}

The electron beam is generated in a $3.2 \mathrm{MV}$ diode. It consists of a thermionic source surrounded by a Pierce electrode and focused by three solenoids located at the anode. A bucking coil is placed close to the source to zero the axial magnetic field in order to minimize the initial canonical angular momentum of the beam; outside the magnetic field this canonical angular momentum would be transformed into beam emittance.

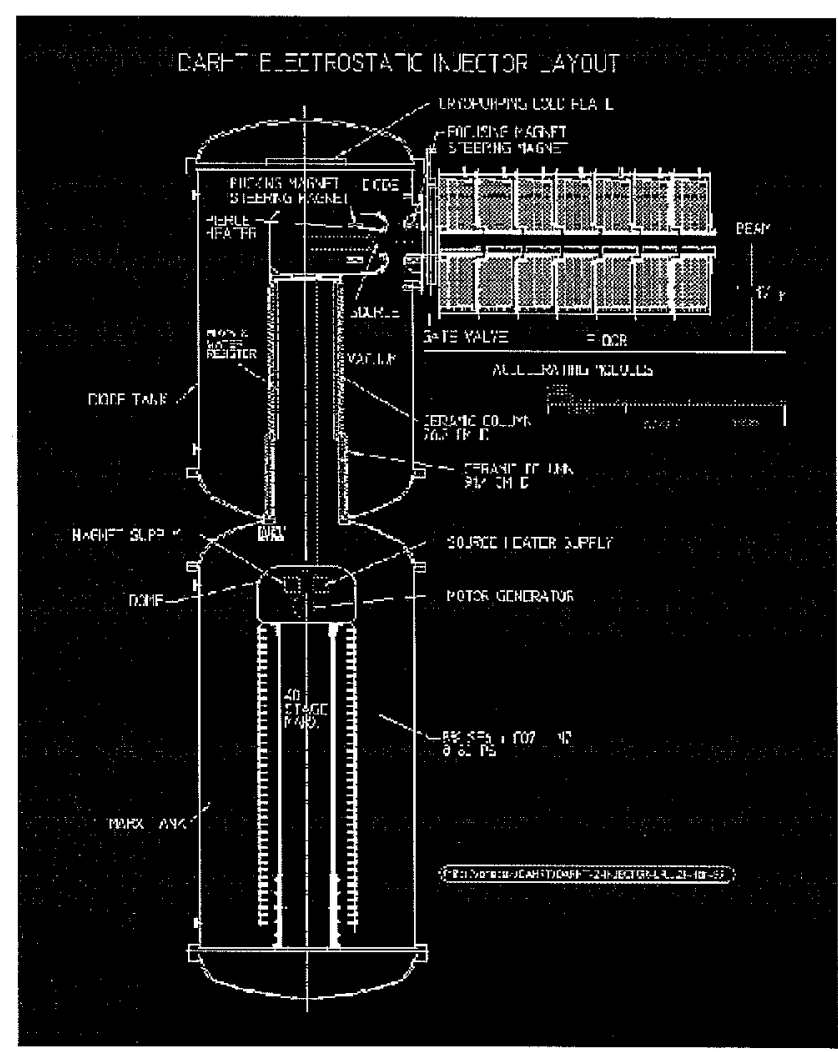

Figure: 1 Main components of a $3.2 \mathrm{MV}$ electrostatic injector.

The beam dynamics inside the diode has been studied using the electron trajectory computer code EGUN [2]. Figure 2 shows the electron beam envelope and field equipotential lines as calculated by EGUN for the 3.2 MV case.

In order to have a reliable machine the diode design has to minimize breakdown risks. This requirement translates into a design with maximum current extraction for a given maximum field stress. From final focus requirements at the end of the machine, the beam quality has to be controlled and the normalized emittance be maintained under $1000 \mathrm{pi}-$ mm-mr.

For a given field stress limit, maximum current extraction is obtained from cathodes surrounded by a flat shroud as compared to diodes incorporating Pierce electrodes. On the other hand, the beam quality is better controlled by a Pierce electrode; flat shrouds produce hollow beams whose normalized emittance grow as being transported and accelerated along the induction linac. A compromise between the two conflicting requirements is to 
design the diode with a Pierce electrode assuming the maximum voltage holding capability that can be obtained using special surface handling procedures.

For the $2 \mathrm{kA}$ case, this peak field is around 120 $\mathrm{kV} / \mathrm{cm}$ on the cathode side of the diode and around 150 $\mathrm{kV} / \mathrm{cm}$ on the anode side. For the $4 \mathrm{kA}$ case the peak field is around $165 \mathrm{kV} / \mathrm{cm}$ on the cathode side of the diode and above $200 \mathrm{kV} / \mathrm{cm}$ on the anode side. For these designs the emittance at the end of the diode is under 1000 pi-mm-mr as calculated by EGUN.

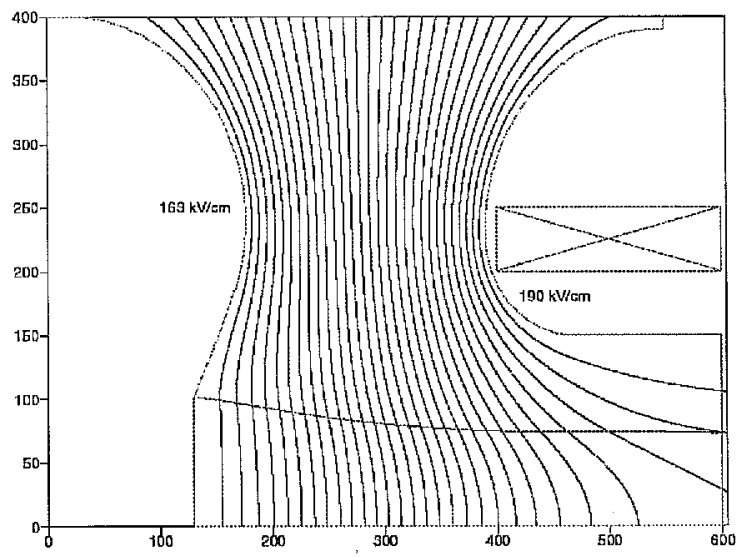

Figure: 2 Electron beam envelope and field equipotential lines as calculated by EGUN..

\section{BEAM DYNAMICS IN THE INJECTOR}

A two-dimensional particle-in-cell (PIC) slice $(x-y)$ code [4] has been used to study the transverse beam dynamics of the electron beam generated at the diode as it is transported and accelerated along the first inductionaccelerator section. This section consists of 8 induction cells each providing $200 \mathrm{kV}$ of acceleration. Each cell contains a solenoid used to focus the electron beam.

Figure 3 shows the beam envelope for various offenergy components (beam-head) during the $400 \mathrm{~ns}$ energy rise time for the $2 \mathrm{kA}$ case. The magnetic tune was chosen to confine the beam envelope within $60 \%$ of the aperture thus avoiding beam spillage on the pipe. Calculation for the $4 \mathrm{kA}$ case show containment of the beam-head within $90 \%$ of the aperture leading to a possibility of beam spillage. To reduce the risk of beam spillage, we are considering shaping the focusing field during the beam rise time by using a time-dependent magnetic field.

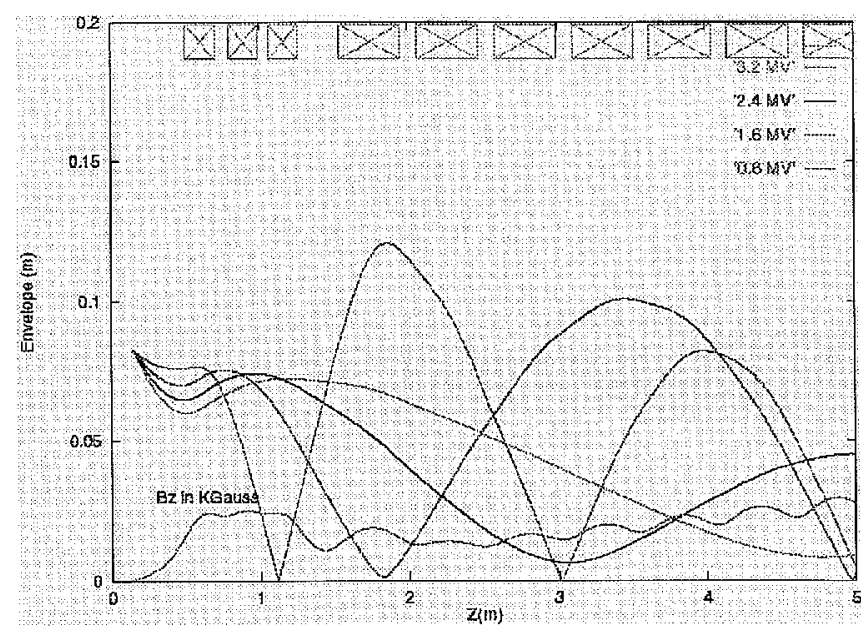

Figure: 3 EGUN simulation of the electron beam from source to the end of the first acceleration section $(7$ cells)

\section{TIME-VARYING MAGNETIC FIELD SOLENOID}

The additional focusing field, $\mathrm{B}_{\mathrm{Z}}(\mathrm{t})$, for the $4 \mathrm{kA}$ case, is applied during the beam rise-time (400ns) to contain the beam envelope at lower energies and eliminate beam spillage. The required $\mathrm{B}_{\mathrm{z}}(\mathrm{t})$ field profile goes down from around 400 Gauss to 0 Gauss within $400 \mathrm{~ns}$, overlapping the beam rise time.

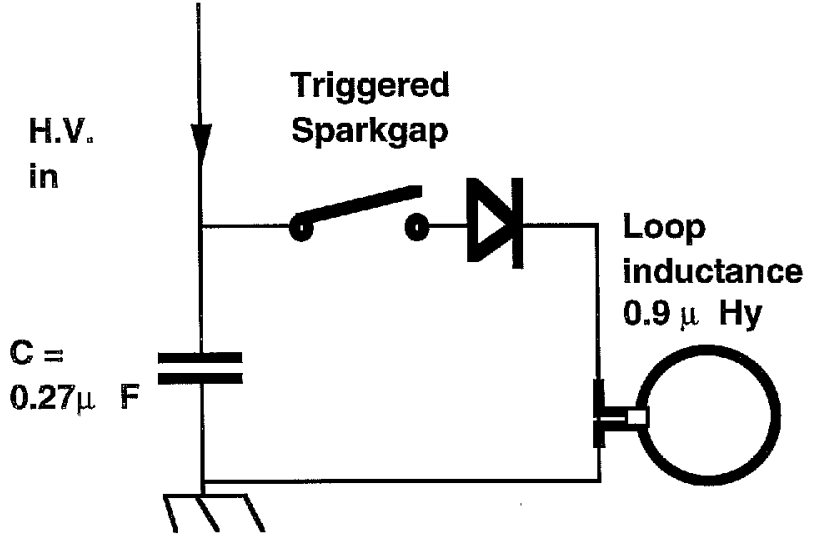

Figure: $4 \quad B_{Z}(t)$ solenoid driver schematics.

The solenoid that generates the time-varying field, which consists of a single loop coil $10.5^{\prime \prime}$ in diameter, is placed in the beam tube under the anode shroud. The solenoid is driven by a simple $6 \mathrm{kA}, 1 \mu \mathrm{s}$ half sine wave current pulse. The solenoid driver (Figure 4) consists of an $\mathrm{LC}$ ringing-discharge circuit. An up to $30 \mathrm{kV}$ spark gap switch is turned-on at $600 \mathrm{~ns}$ before the beam turn-on time. The current waveform (Figure 5) will be limited to a half cycle, so that it will not interfere with the main body of the beam, by using high current $(1.5 \mathrm{kA})$, high voltage $(10 \mathrm{kV})$ semiconductor diodes. We measured the effect of the beam tube walls on the $\mathrm{B}_{\mathrm{z}}(\mathrm{t})$ for various solenoid diameters. 
Following the measurements results we limited the solenoid diameter to $60 \%$ of the beam tube diameter where the tube wall effect on $B_{z}(t)$ is negligibe.

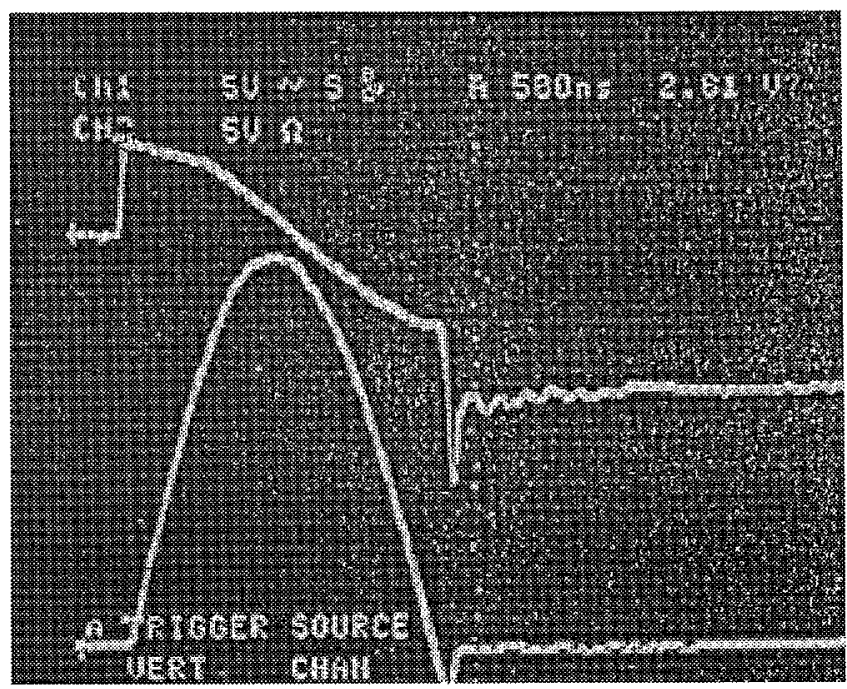

Figure: 5 Waveform of the $3 \mathrm{kA}$ current (bottom curve) driving the time-varying solenoid.

\section{DIODE DESIGN SUPPORT EXPERIMENTS}

Scaled experiments are under construction to support the DARHT-II injector design.

The LBNL RTA 1MV, 1kA, 300 ns injector [3] diode configuration allows the extraction of a high quality, high density beam. The injector AK gap, cathode shroud material and shape were designed to allow the study of vacuum breakdown issues in the diode in the presence of a beam.

The RTA thermionic source heat management and support stability were tested. Thermocouples can be placed along the source support to map the temperature and evaluate flow in the cathode assembly. Furthermore the gap between the source and cathode shroud was monitored during the source heating experiment. This gap has to be small $(<.5 \mathrm{~mm})$ to insure a high quality beam. Initial measurements using a hot wire pyrometer, performed on the RTA 3.5" diameter source at a cathode surface temperature of $1060^{\circ} \mathrm{C}$ showed a temperature uniformity within $5^{\circ} \mathrm{C}$. A temperature of $30^{\circ} \mathrm{C}$ was measured using a thermocouple at the cathode assembly input flange showing a good heat management. A gap around 24 mils between the source surface and the cathode shroud was observed using a survey telescope looking at the source through a front window.

Initial breakdown experiments were performed with a long duration ( $8 \mu$ s discharge time constant) $200 \mathrm{kV}, 0.5 \mathrm{~kJ}$ pulser. Using machine-polished stainless steel, molybdenum and copper electrodes we measured breakdown fields above $400 \mathrm{kV} / \mathrm{cm}$ in a $3 \mathrm{~mm}$ gap. Figure 3 shows that the breakdown field is independent on vacuum pressure within a rang of $10-(8)$ to 10 -(4) Torr. We observed more damage on the copper and stainless steel electrodes and no difference in the breakdown voltage between stainless steel, copper and molybdenum electrodes.

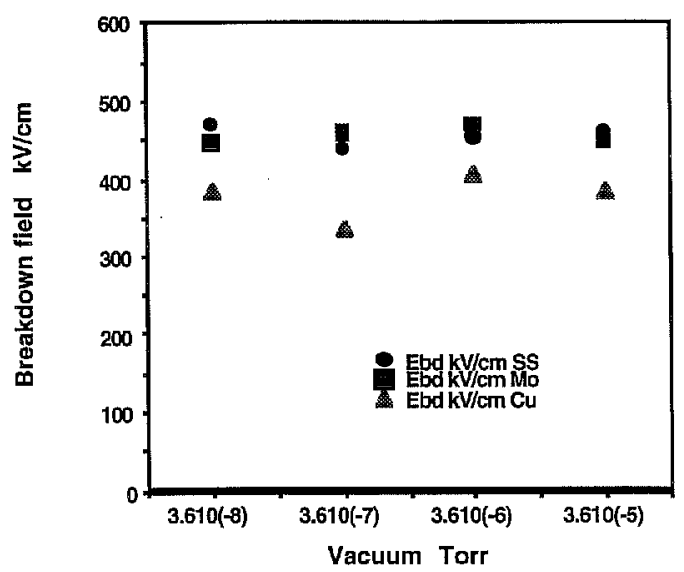

Figure: 6 Breakdown field dependence on vacuum pressure and electrode material.

\section{ACKNOWLEDGEMENTS}

This work was performed under the auspices of the U.S. Department of Energy under contract AC0376SF00098. We thank Dr. D. Spreyn and his colleagues at SLAC for fruitful discussions on breakdown issues.

\section{REFERENCES}

[1] E Henestroza, "Injectors for Heavy Ion Fusion", invited talk, 11th International Workshop on Laser Interaction and Related Plasma Phenomena, Monterey, CA, October 25-29, 1993. AIP Conference Proceedings 318, Ed. G.H. Miley, pp 577-582.

[2] W.B. Herrmannsfeldt, "EGUN- An electron optics and gun design program," SLAC-Report-331, 1988.

[3] S. Lidia et al., "Initial Commissioning Results of the RTA Injector", this conference.

[3] B. Carlsten, "The PIC-xy 2-D SLICE code", private communication. 